developed case of pediculosis corporis, including those that separate it from Addison's disease. He was not in perfect health, certainly, but he was not cachectic, and had latterly been improving; so that, except for one fact, nothing could authorise a diagnosis of disease of the adrenals. The one fact, pointing the other way, was that the mucosa of the buccal cavity was the seat of a true pigmentation, in the form of rounded patches, 3 or $4 \mathrm{~mm}$. in diameter. M. Thibierge's second case possessed the same features.

M. Chauffard said that some time ago he had seen a patient, who proved to be phthisical, and who had a very peculiar colour of skin, with pigmented areas on the buccal mucous membrane. M. Chauffard had therefore concluded that this was a case of Addison's disease. When the patient died, however, the autopsy showed that the supra-renal capsules were perfectly healthy; and as pediculosis had long been present, M. Chauffard now felt inclined to place the case alongside those which had just been related by M. Thibierge. (La France Médicale.)

\title{
DISEASES OF THE SKIN.
}

\section{BY Dr. A. NAPIER.}

Chromic Acid in Syphilitic Ulceration.-Dr. Ernest Feibes confirms the great value of this remedy, as pointed out by Schuster, Vidal, Butlin, and others. He states that he always uses chromic acid in the local treatment of syphilides of the mucous membrane, and with results to be obtained by no other methods, so that the application of the nitrate of silver point falls more and more into disuse. A case is quoted of a man who had already been treated for several weeks, on account of specific ulcers of the tongue, with mercurial pills and the local application of nitrate of silver. The result not being satisfactory, the patient was put through a course of inunction, and the parts touched with the silver point. No change being perceptible within ten days, a solution of chromic acid (one to two) was used locally. After the application the ulcer was seen covered with a yellow pellicle, which separated in two days, showing the affected part much smaller; and complete healing occurred within eight days, the ulcer being touched every second day. Ten cases of broken-down gummatous nodules of the tongue were treated with chromic acid and did extremely well, healing in a much shorter time than with the nitrate of silver treatment. The application is but slightly painful, though the taste is very objectionable. Mucous patches are rapidly removed by the chromic acid solution. Feibes has repeatedly seen them disappear in two to three days. A similar result is obtained in cracks around the angle of the mouth. One of the most obstinate forms of syphilis of the mouth, the specific lingua geographica, is affected by no treatment so readily as by this. In such a case, the tongue was carefully dried with cotton wool, and so isolated, after which concentrated chromic acid solution was applied by means of a brush, allowed to remain on for some minutes, and then washed off with acetate of aluminium solution. In three days, the necrotic tissue had separated, displaying the normal looking tongue. Five such applications within fourteen days sufficed for a complete cure. Eleven cases of lingual psoriasis were likewise treated satisfactorily. When warty irregularities were present they were first scraped with the sharp spoon, and when the bleeding had ceased, touched with chromic acid. Finally Feibes has found the acid very useful in mercurial stomatitis. Here a fine sound, carrying cotton-wool, is introduced between the tooth and the gum, and the foul matter carefully removed. Another sound dipped in concentrated chromic acid solution is then inserted between the gum and tooth, and the patient directed to use acidulated chloroform water on account of the disagreeable taste. This method has yielded most excellent results.-(Therapeutische Monatshefte, No. 11, 1891 ; Practitioner, p. 126, February, 1892.) 
Bony Deposits in the Skin.-Mr. Faulder White read a paper on this subject before the Birmingham. and Midland Counties Branch of ,the British Medical Association. He first quoted the case described by Mr. Jonathan Hutchinson in the Medical Times for 1860, and then recounted the following case met with in his own practice:-Col. C., aged 65, met with a railway accident in 1842. The skin of his legs was almost completely destroyed by the steam from the burst boiler. The process of healing occupied five years. During the ensuing quarter of a century the cicatrices had "time to wear out," the new skin becoming generally movable over the underlying structures. During the next twenty years a development took place of bony masses in the deeper layers of the skin. At one time a bony mass encircled the left leg. Plates formed in each calf and in front of the knee joint. The removal of some plates became necessary on account of irritation, causing acute dermatitis or sloughing ulcers. Patches of ichthyosis formed below the knees, proving that ichthyosis is not always congenital, as Morris states it probably is. Mr. White said that the bony plates formed part of the tissues in which they lay, and appeared to have originated in an actual transformation of these tissues. To the naked eye they presented all the features of true bone; microscopically there were no typical Haversian systems, but glistening homogeneous lamellæ. On the other hand none of the usual appearances of granular degeneration were seen. Mr. White quoted some facts which lead to the belief that the connective-tissue cell may be an important agent in the process of calcification. He referred to the belief that in all cases of calcification there exists some local impediment to the circulation. In regard to the bony plates-the subject of his paper-he submitted that they were the result of the simultaneous action of the process of deposition and incorporation, the least soluble salts of the nutritive fluids being left by a particularly sluggish current to become subject to the selective activity of the connective-tissue cell.--(The Birmingham Med. Review, p. 289, vol. ii, 1891 ; Practitioner, p. 132, February, 1892.)

Alkalies in Universal Pruritus. - Lange (in Jour. of Cut. and GenitoUrin. Dis., October, 1891), on the basis of four cases, recommends such alkalies as sodium bicarbonate and lithium carbonate in the treatment of universal pruritus. In one case, that of a lady of 51, the itching had localised itself in the genital region, and was so extremely severe, that the patient seemed threatened with actual loss of reason with suicidal impulses. Ultimately she became very emaciated, and the itching spread over the entire surface, and sleep was obtained only through large doses of chloral, and the local application of very hot compresses. She recovered completely under the alkaline treatment, and the local use of carbolic acid compresses. As a general indication for such treatment, the author speaks of finding abundant deposits of uric acid and urates in the urine.

Skin Eruptions in Uræmia.-In the British Medical Journal, p. 1099, vol. ii, 1891, Dr. Le C. Lancaster draws attention to an eruption which occurs most commonly in cases of chronic interstitial nephritis, and which must be regarded as a grave prognostic indication-seven out of eight patients in whom it occurred died within five weeks of its appearance. It takes the form at first of bright red maculæ or papulæ on the extensor surface of hands, forearms, and legs, spreading very soon, however, so as to involve the whole body. Within a few days the eruption may fade, being followed by copious desquamation, while the skin is left thick and brawny; or the eruption may assume an eczematous form, with exudation of a sticky fluid and the formation of scabs and crusts ; or, as in the graver cases, pustulation may occur, or even the formation of small abscesses, as a sequel to the eczematous stage. Throughout the whole course of the skin affection there is severe itching. 